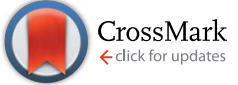

Cite this: Chem. Sci., 2017, 8, 577

\title{
Organic solid fluorophores regulated by subtle structure modification: color-tunable and aggregation-induced emission $\dagger$
}

\author{
Jing Nan Zhang, $\dot{t}^{a}$ Hui Kang,,$^{a}$ Nan Li, ${ }^{a}$ Shi Ming Zhou, ${ }^{c}$ Hua Ming Sun, ${ }^{a}$ Shi Wei Yin, ${ }^{a}$ \\ $\mathrm{Na}$ Zhao $^{\star a}$ and Ben Zhong Tang ${ }^{\star b}$
}

Organic solid fluorophores with a tunable emission color have been widely applied in multiple areas. However, rational design and efficient crafting of these fluorophores from a simple core skeleton is still a formidable challenge because of the undesirable concentration quenching emission effect. Herein, we present the development of two series of organic solid fluorophores based on a backbone of $p$-bis(2,2-dicyanovinyl)benzene. Notably, the introduction of either non-aromatic or aromatic substituents provides fluorophores with a tunable emission color. Moreover, the fluorophores with aromatic substituents exhibit additional unique optical phenomena, such as aggregation-induced emission, polymorphism-dependent emission, and reversible mechanochromic luminescence.

Received 29th June 2016

DOI: $10.1039 / \mathrm{c} 6 s c 02875 f$

www.rsc.org/chemicalscience

the ACQ process. ${ }^{6,7}$ To date, numerous elegant organic solid emitters based on diverse core skeletons have emerged that exhibit the AIE effect, and extensive technological applications have been developed. ${ }^{8}$ However, AIE systems with a tunable emission color that are based on a simple core backbone are still extremely rare. ${ }^{9}$

Recently, extended $\pi$-conjugated molecules with a D- $\pi-\mathrm{A}$ structure have attracted significant interest because of their flexibility for creating organic emitters with tunable emission. ${ }^{\mathbf{1 0} 11}$ The nitrile group, due to its strong electron-withdrawing ability and structural simplicity, is an ideal candidate for an excellent acceptor for such $D-\pi-A$ fluorescent materials featuring advanced photoluminescence properties. More importantly, the nitrile group can induce steric hindrance and result in a twisted conformation, which enables the fluorophores to be protected from the ACQ effect. ${ }^{12}$ However, studies on regulating the emission behavior of a nitrile-containing system through altering the substituents are rare. ${ }^{9}$ Herein, we present the development of two series of symmetric $p$-bis(2,2-dicyanovinyl)benzenebased fluorophores by decorating the single benzene ring with a dicyanovinyl unit as the acceptor and different types of donors with diverse electronic and steric effects (Fig. 1). Interestingly, with the introduction of either non-aromatic or aromatic substituents, both classes of fluorophores yield a full-color emission. Moreover, the aromatic groups can successfully endow the fluorophores with a highly efficient solid state emission and AIE effect. Meanwhile, a specific polymorphism-dependent emission as well as reversible mechanochromic luminescence are observed for some of the AIE fluorophores. 


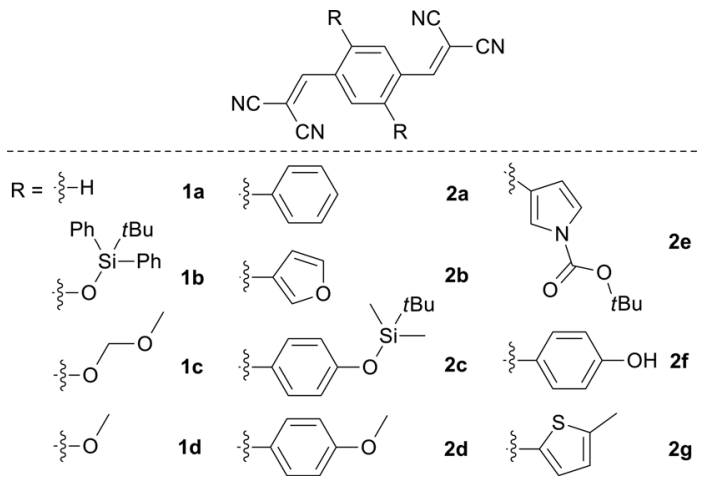

Fig. 1 Chemical structures of $1 \mathrm{a}-2 \mathrm{~g}$.

\section{Results and discussion}

The initial step of our research was to merge the components of both the donor and acceptor to form the desired compounds 1a-2g. This was readily achieved using a one-step condensation reaction between the appropriate aldehyde and malononitrile. All the compounds were easily purified using column chromatography or recrystallization with a reasonable yield.

The absorption and photoluminescence properties of all the compounds in acetone and the solid state are shown in Table 1, as well as Fig. S1 and S2. $\dagger$ For the parent compound 1a, the absorption peak with the longest wavelength was located at around $367 \mathrm{~nm}$, which was ascribed to the $\pi-\pi^{*}$ transition. When the substituent group at the 2,5-positions was altered from $t$-butyldiphenylsiloxyl to methoxyl (or from aromatic phenyl to 5-methylthienyl for series 2), the corresponding compounds $\mathbf{1 b} \mathbf{b} \mathbf{1 d}$ (or 2a-2g) exhibited the longest wavelength absorption peak ranging from 428 to $457 \mathrm{~nm}$ (or 392 to $441 \mathrm{~nm}$ ), which was assigned to the intramolecular charge transfer (ICT) transition from the different type of electron-donating group to the electron-accepting dicyanovinyl group. ${ }^{10}$
As shown in Table 1, with increase of the electron-donating ability, the emission wavelengths of $\mathbf{1 a - 1 d}$ in acetone presented a red-shift from 426 to $562 \mathrm{~nm}$, while the emission peak wavelength for the solid state ranged from 467 to $620 \mathrm{~nm}$. By changing the aromatic group substituent, the emission peaks of $2 \mathrm{a}-2 \mathrm{~g}$ in acetone varied from 495 to $600 \mathrm{~nm}$. Additionally, a similar red shifted emission tendency (464 to $622 \mathrm{~nm}$ ) for the solid state was observed. It is clear that two series of solid emitters with emission over the entire color range were obtained by simply varying the type of donor (Fig. 2). The CIE chromaticity diagrams (Fig. S3 and S4, Table S3†) demonstrated the same tunable emission from CIE blue $(0.1374$, $0.0374)$ to CIE red $(0.6954,0.3045)$. Furthermore, single crystals for all the compounds, except $\mathbf{1 b}$ and $\mathbf{2 f}$, were obtained by slow evaporation from an appropriate solvent (Tables S6-S8, Fig. S5 $\dagger),{ }^{13}$ and they also emitted fluorescence covering the whole visible region (464 to $640 \mathrm{~nm}$ for series 1 and 467 to $593 \mathrm{~nm}$ for series 2 ).
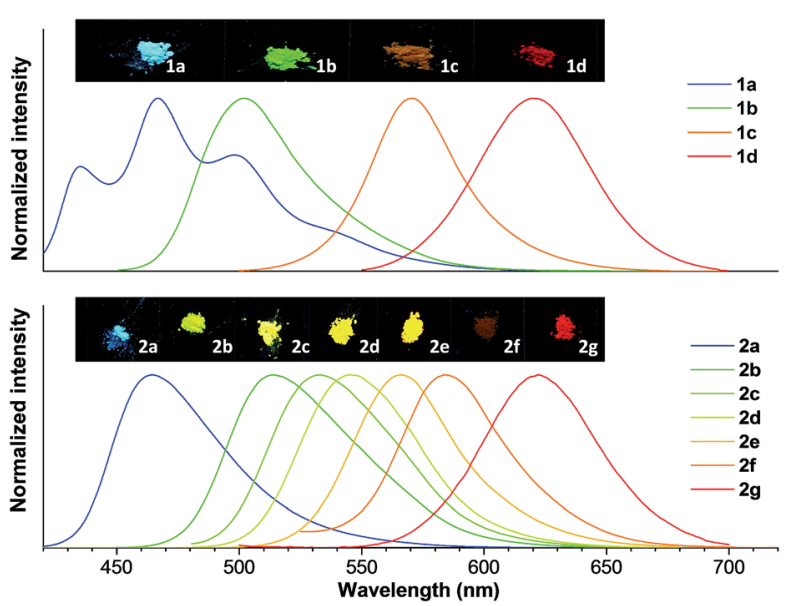

Fig. 2 Normalized PL spectra of $1 a-1 d$ and $2 a-2 g$ in the solid state, and photographs taken under irradiation with $365 \mathrm{~nm}$ UV light.

Table 1 Optical properties of $1 \mathrm{a}-2 \mathrm{~g}$

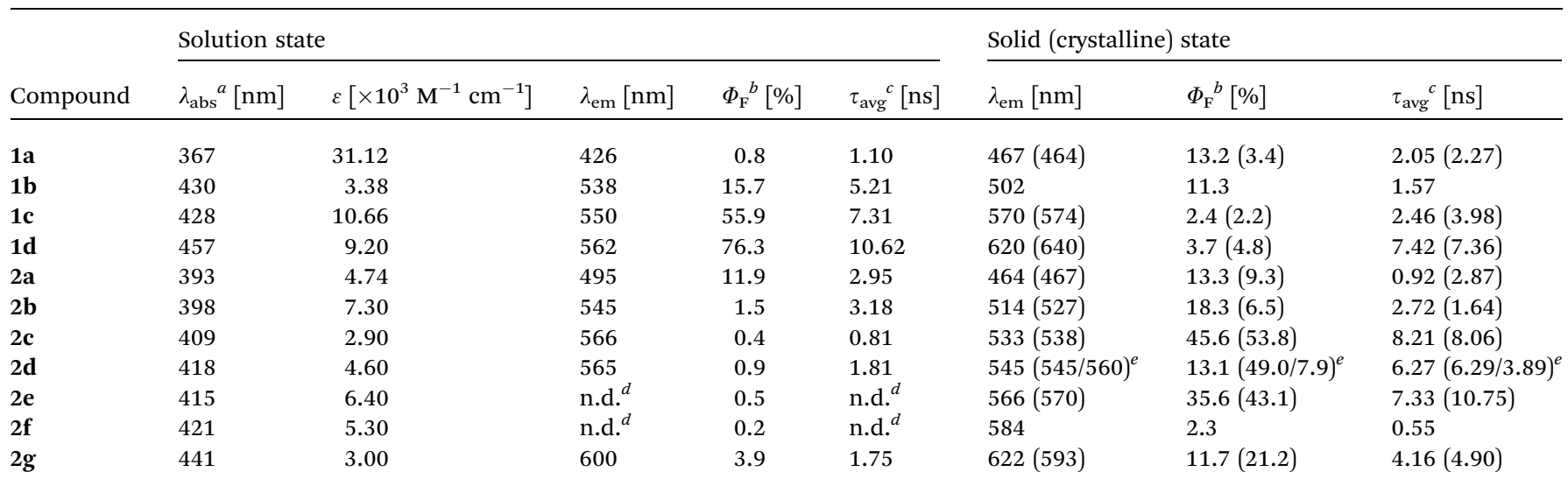

${ }^{a}$ Longest wavelength absorption maximum in acetone. ${ }^{b}$ Absolute fluorescence quantum yield obtained using the calibrated integrating sphere system. ${ }^{c}$ Mean fluorescence lifetime $\left(\tau_{\text {avg }}\right)$ calculated using the equation $\tau_{\text {avg }}=A_{1} \tau_{1}+A_{2} \tau_{2} .{ }^{d}$ Below the detection limit. ${ }^{e}$ Two types $(\mathbf{2 d - g} / \mathbf{2 d - o})$ of crystal 2d. 
To better understand the electronic transitions of all the compounds, density functional theory (DFT) calculations, using the single crystal structure determined from X-ray analysis, were carried out (Table S1†). The results indicated that both the highest occupied molecular orbital (HOMO) and the lowest unoccupied molecular orbital (LUMO) of 1a were located over the whole molecular framework, suggesting a $\pi-\pi^{*}$ transition. For the other compounds, however, the HOMO was mainly located on the core benzene ring and the electron-donating moieties, while the LUMO was spread primarily over the central benzene ring and the electron-withdrawing dicyanovinyl group, which demonstrated the existence of ICT character for those compounds. Meanwhile, the HOMO and LUMO became more separated in series 2 , compared with series $\mathbf{1}$, which was ascribed to the twisted conformation of series 2 originating from steric hindrance due to the introduction of the bulky aromatic group.

The dipole moment change $(\Delta \mu)$ between the excited state $\left(\mu_{\mathrm{e}}\right)$ and ground state $\left(\mu_{\mathrm{g}}\right)$ was also determined (Table S $\left.2 \dagger\right)$. The results showed that the $\Delta \mu$ values for series 2 were larger than for series $\mathbf{1}$, which is in agreement with the degree of separation between the HOMO and LUMO. Furthermore, 1b-2g exhibited obvious solvent effects (Fig. S6 and $\mathrm{S} 7 \dagger$ ). ${ }^{\mathbf{1 4}}$ With increase of the solvent polarity (from cyclohexane to acetone or dichloromethane), the emission peak exhibited a red-shift, which indicated the existence of ICT in these compounds. However, a slight solvent effect was observed for 1a, confirming its $\pi-\pi^{*}$ character.

The fluorescence quantum yields $\left(\Phi_{\mathrm{F}}\right)$ of all the compounds in solution and the solid state (including the crystalline state) are also summarized in Table 1 . The quantum yields of $\mathbf{1 b} \mathbf{b} \mathbf{1 d}$ dissolved in acetone were moderate to high (15.7-76.3\%), but significantly decreased for the solid state $(<11.3 \%$ for the solid powder, $<4.81 \%$ for the crystal). In sharp contrast, a dramatic enhancement was achieved for $\mathbf{1 a}$ and $\mathbf{2 a}-\mathbf{2} \mathbf{g}$ in the solid state $(2.3-45.6 \%$ for the solid powder, $3.4-58.8 \%$ for the crystal) compared with in solution (0.2-11.9\%). It is noteworthy that 1d and $2 \mathbf{g}$ exhibited red emission. More importantly, $\mathbf{2} \mathbf{g}$ retained a high quantum yield in the solid state $(622 \mathrm{~nm}, 11.7 \%)$ and crystalline state $(593 \mathrm{~nm}, 21.2 \%)$. Considering that red fluorophores containing $\pi$-conjugated frameworks typically have a tendency to aggregate in the solid form, which results in emission quenching, this strategy provides an extremely powerful method to fabricate organic solid emitters with a long emission wavelength. We also calculated the ratio between the solid and solution state quantum yields to verify the influence of the substituent group on the emission behavior of the compounds (Fig. 3D). It is clear that the ratio was greater than one for $\mathbf{1 a}$ and $\mathbf{2} \mathbf{a}-\mathbf{2} \mathbf{g}$ but less than one for $\mathbf{1 b} \mathbf{b} \mathbf{1 d}$. Therefore, the introduction of an aromatic group successfully reversed the trend of the quantum yields between the solution and solid phases.

A higher quantum yield for the solid state implies existence of the AIE effect. Therefore, we measured the AIE properties using acetone and water, which served as a good and poor solvent, respectively. Typically, 2e was almost non-emissive when dissolved in acetone. Increase of the water fraction $\left(f_{\mathrm{w}}\right)$
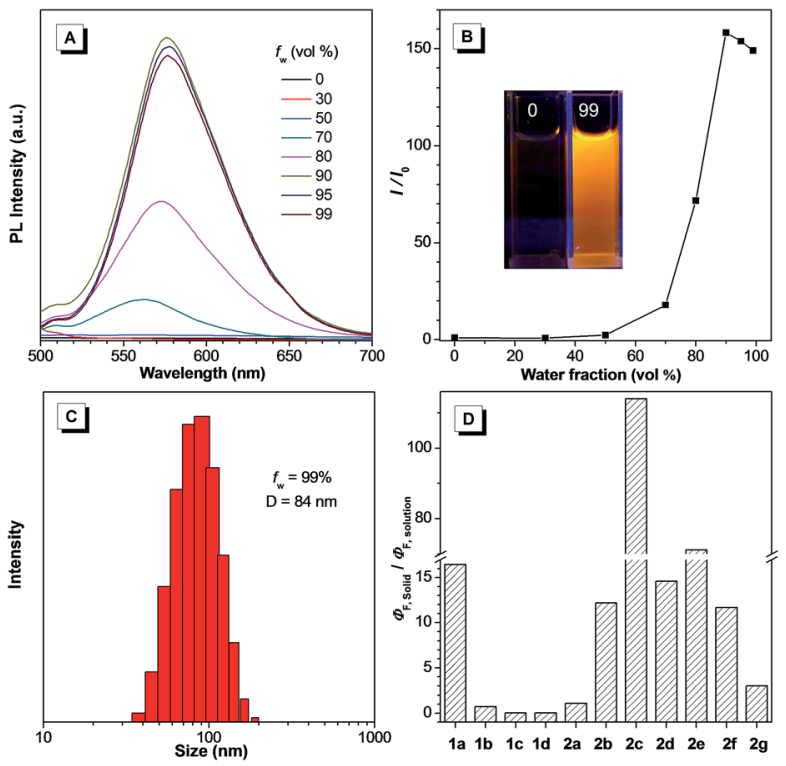

Fig. 3 (A) PL spectra of $2 \mathrm{e}(10 \mu \mathrm{M})$ in acetone and acetone/water mixtures with different water fractions $\left(f_{\mathrm{w}}\right)$. (B) Plot of the emission intensity versus the composition of the water mixture for $2 \mathrm{e}$. (C) Particle size distribution of $2 \mathrm{e}(10 \mu \mathrm{M})$ in an acetone/water mixture with a $f_{w}$ value of $99 \%$. (D) The ratio of the quantum yields for the solid and solution states of all the compounds. Inset in (B): photographs of $2 \mathrm{e}$ in acetone/water mixtures with $f_{\mathrm{w}}$ values of 0 and $99 \%$ under irradiation with 365 nm UV light.

resulted in a significant boost of the emission intensity at $576 \mathrm{~nm}$, and yielded approximately a 150-fold enhancement when the $f_{\mathrm{w}}$ was increased up to $99 \%$ (Fig. 3A and $\mathrm{B}$ ). The observed phenomenon could be attributed to aggregation of the molecules in the mixed solution with a high $f_{\mathrm{w}}$, and thus activation of the RIR process. The particle size for $2 \mathbf{e}$ in the mixture with a $f_{\mathrm{w}}$ of $99 \%$ was determined to be $84 \mathrm{~nm}$ using dynamic light scattering (DLS), confirming the formation of nanoaggregates (Fig. 3C). Furthermore, the other compounds in series 2 and 1a also displayed a similar AIE or aggregationinduced emission enhancement (AIEE) performance (Fig. S8, S12-S17†). Nevertheless, compounds 1b-1d with non-aromatic moieties exhibited an opposite phenomenon, where increase of the water fraction resulted in a gradual decrease of the emission intensity, suggesting ACQ properties (Fig. S9-S11†).

The fluorescence lifetime $(\tau)$ values of all the compounds are listed in Tables 1 and $S 4 . \dagger$ To verify the photoemission dynamics, the radiative transition rate constant $\left(k_{\mathrm{r}}\right)$ as well as the nonradiative transition rate constant $\left(k_{\mathrm{nr}}\right)$ were calculated using the fluorescence lifetimes and the quantum yields (Table S5 $\dagger$ ). Taking $1 \mathrm{~d}$ as an example, the $k_{\mathrm{nr}}\left(2.232 \times 10^{7} \mathrm{~s}^{-1}\right)$ was close to the $k_{\mathrm{r}}\left(7.185 \times 10^{7} \mathrm{~s}^{-1}\right)$ in solution. However, the $k_{\mathrm{nr}}$ was enhanced to $1.297 \times 10^{8} \mathrm{~s}^{-1}$ while the $k_{\mathrm{r}}$ was reduced to $4.986 \times 10^{6} \mathrm{~s}^{-1}$ in the solid state, implying that nonradiative decay was dominant in the solid state. Whereas for $2 \mathrm{c}$, the $k_{\mathrm{nr}}\left(1.229 \times 10^{9} \mathrm{~s}^{-1}\right)$ was remarkably greater than the $k_{\mathrm{r}}$ $\left(4.938 \times 10^{6} \mathrm{~s}^{-1}\right)$ in solution. However, the $k_{\mathrm{nr}}$ was decreased to $6.626 \times 10^{7} \mathrm{~s}^{-1}$ while the $k_{\mathrm{r}}$ was enhanced to $5.554 \times 10^{7} \mathrm{~s}^{-1}$ in the solid state. These results suggest that suppression of the 
nonradiative decay is the major reason for the higher quantum yield in the solid state.

To obtain the underlying reasons for this significant substituent dependent emission behavior, inspection of the single crystals was performed. Fig. 4 presents the single crystals of $1 \mathbf{d}$ and 2c as examples. Crystal 1d adopted a near-planar conformation and the torsion angle between the vinyl (or methyl) group and the central benzene ring was merely $3.42^{\circ}$ (or $3.63^{\circ}$ ). Careful evaluation of the details of the crystal packing indicated that the distance between two adjacent planes was 3.416 $\AA$, which suggested that 1d encounters strong and consecutive intermolecular $\pi-\pi$ stacking interactions and thus this resulted in weakening of the fluorescence in the solid state (Fig. 4A) ${ }^{15}$ Similar strong $\pi-\pi$ stacking interactions (3.214 $\mathrm{\AA}$ ) were also observed for the crystal of 1c (Fig. S21B $\dagger$ ), and were responsible for the reduced fluorescence in the solid state. In contrast, the crystal of $2 \mathrm{c}$ clearly showed a contorted conformation, where the torsion angle between the vinyl (or substituted phenyl) group and the central benzene ring was $42.14^{\circ}$ (or $40.15^{\circ}$ ), which apparently resulted from steric congestion between the neighboring phenyl rings and dicyanovinyl moieties. Such a twisted conformation effectively enlarged the distance between the adjacent phenyl groups (>4.076 $\AA$ ), and avoided the typical $\pi-\pi$ stacking interactions (Fig. 4B). Meanwhile, crystallographic analyses of the other compounds in series 2 showed similar twisted conformations and an absence of $\pi-\pi$ stacking interactions, which accounts for their bright luminescence in the solid state (Fig. S22-S24†). Compared with the other molecular packing arrangements in series 2 , it is clear that introduction of a bulky substituent did not enlarge the distance between adjacent molecules, implying a relatively tight packing in the crystals of $2 \mathbf{c}$ and 2e. Additionally, multiple intermolecular $\mathrm{C}-\mathrm{H} \cdots \mathrm{N}$ (or $\mathrm{C}-\mathrm{H} \cdots \mathrm{O}$ ) and $\mathrm{C}-\mathrm{H} \cdots \pi$ interactions were found throughout the crystal structures of 2c and 2e (Fig. S25 and S23A $\dagger$ ), which could help to rigidify the molecular conformation, and hence result in their relatively high emission efficiency in the solid state. It is interesting to note that there were also no $\pi-\pi$ stacking interactions in the crystal of 1a,

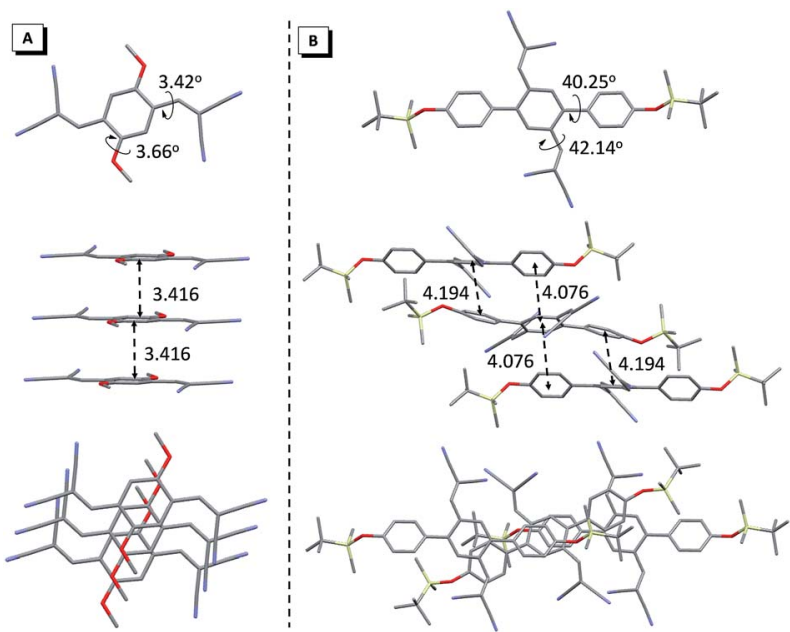

Fig. 4 Single crystal structures of $1 d(A)$ and $2 c(B)$, and the molecular packing viewed from the side and top of adjacent molecules. though it exhibited an almost planar conformation (Fig. S21 $\mathrm{A}_{\dagger}^{\dagger}$ ), which led to no emission quenching in the solid state.

Unexpectedly, two types of $\mathbf{2 d}$ crystals, with crystallographically independent conformations (2d-g and 2d-o), suitable for Xray analysis were obtained from ethyl acetate/dichloromethane and ethyl acetate/acetone mixtures. The crystal $\mathbf{2 d - g}$ exhibited a green emission at around $545 \mathrm{~nm}$, while $2 \mathrm{~d}$-o showed a bathochromic shift with an orange emission at $560 \mathrm{~nm}$ (Fig. 5A). Both 2d-g and 2d-o adopted highly twisted conformations without $\pi-\pi$ stacking interactions (Fig. S24†). The most pronounced difference between the two forms was in the dihedral angle between the dicyanovinyl unit and 4-methoxyl phenyl moiety, which was $46.27^{\circ}$ for $2 \mathbf{d}-\mathbf{g}$ and $43.06^{\circ}$ for $\mathbf{2 d - o}$, respectively (Fig. 5B). This difference indicated that the conformation of $\mathbf{2 d - o}$ was more planar than that of $\mathbf{2 d - g}$, thus this endowed 2d-o with a longer emission wavelength. Moreover, the two crystals presented entirely different packing modes (Fig. 5C and D) and the amount of intermolecular $\mathrm{C}-\mathrm{H} \cdots$ $\mathrm{N}$ interactions between the cyano and phenyl or methyl groups in $\mathbf{2 d - g}$ was more than in $\mathbf{2 d - o}$ (Fig. S24†), enabling $\mathbf{2 d - g}$ to possess a higher quantum yield.

It is notable that the as-prepared sample $\mathbf{2 d}$ exhibited an emission peak at $545 \mathrm{~nm}$, which is close to that of $\mathbf{2 d - g}$. However, gentle grinding induced a red-shift of the emission to $567 \mathrm{~nm}$, which could be ascribed to an amorphous form of $2 \mathrm{~d}$ (Fig. 6A). Such an amorphous state was further confirmed by the flat signal obtained from powder XRD measurement (Fig. S20B $\dagger$ ). Meanwhile, the red-shifted emission could be attributed to collapse of the crystalline lattice during grinding, which would result in the molecules adopting a more planar conformation. An apparent phenomenon is that an amorphous solid can always be crystallized by either heating or fuming with an organic solvent vapor. ${ }^{16}$ Indeed, this was also applicable for
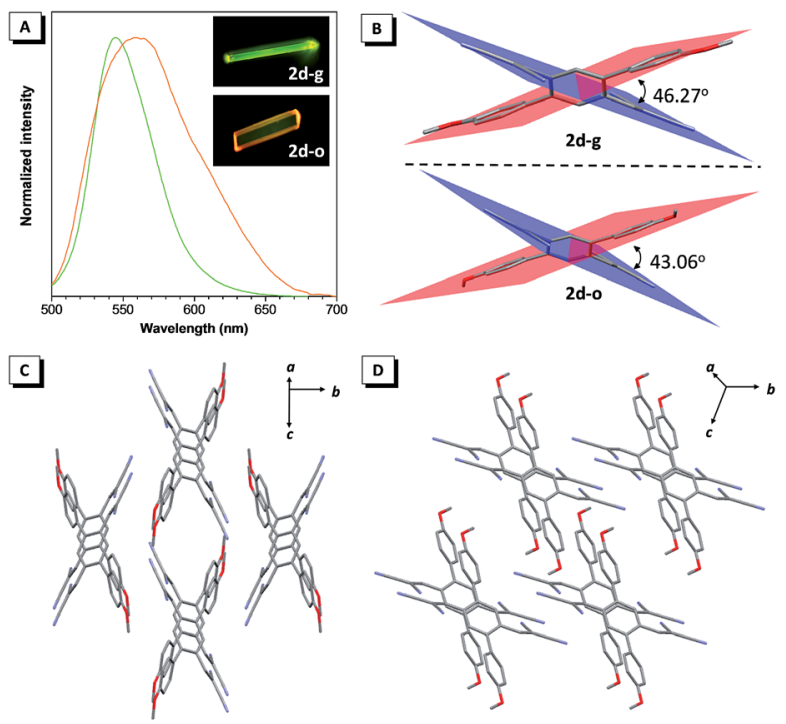

Fig. 5 (A) PL spectra of $2 \mathrm{~d}-\mathrm{g}$ and $2 \mathrm{~d}-\mathrm{o}$. (B) A view of the dihedral angles for $2 \mathrm{~d}-\mathrm{g}$ and $2 \mathrm{~d}-\mathrm{o}$. Molecular packing in $2 \mathrm{~d}-\mathrm{g}(\mathrm{C})$ and $2 \mathrm{~d}-\mathrm{o}(\mathrm{D})$. Inset in (A): photographs of $2 \mathrm{~d}-\mathrm{g}$ and $2 \mathrm{~d}-\mathrm{o}$ under a fluorescence microscope. 

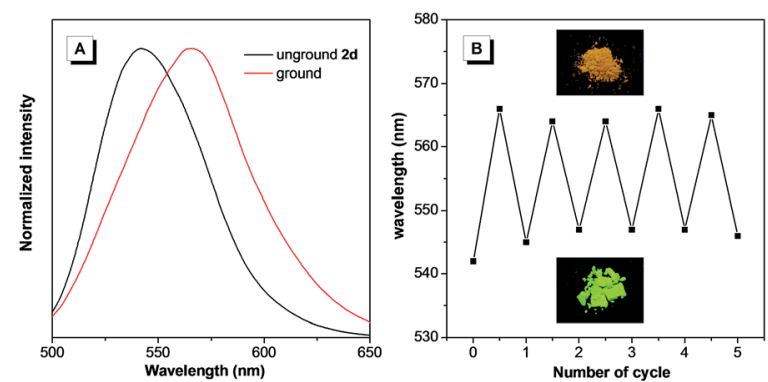

Fig. 6 (A) PL spectra of unground and ground 2d. (B) Reversible switching of the emission of $\mathbf{2 d}$ through repeated grinding/fuming cycles. Inset in (B): photographs of the as-prepared $2 \mathrm{~d}$ and after grinding, under irradiation with $365 \mathrm{~nm}$ UV light.

2d (Fig. 6B). On fuming with a dichloromethane vapor, the amorphous form of $\mathbf{2 d}$ converted into a crystalline form with the emission peak restored to $545 \mathrm{~nm}$. Moreover, this process could be repeated several times, indicating the reversible mechanochromic luminescence properties of $\mathbf{2 d}$ in response to external stimuli. Similar reversible emission behavior for as-prepared powders of $\mathbf{2 b}$ and $\mathbf{2 c}$ could also be achieved through a repeated grinding/solvent fuming process (Fig. S18 and S19†).

\section{Conclusions}

In conclusion, two classes of $p$-bis(2,2-dicyanovinyl)benzenebased fluorophores were designed and synthesized. Their optical properties, as well as their single crystal packing structures, were systematically investigated. The results showed that decorating the core backbone with either non-aromatic or aromatic groups generated two kinds of organic solid emitters with tunable emission from the blue to red region. More importantly, the employment of bulky aromatic groups successfully resulted in a twisted molecular conformation. Such a contorted conformation is beneficial for the inhibition of detrimental $\pi-\pi$ stacking interactions and assisted the fluorophores to exhibit remarkable AIE features with a high emission efficiency in the solid state. In addition, some AIE fluorophores presented polymorphism-dependent emission and reversible mechanochromic luminescence properties. Since these fluorophores exhibiting diverse emission characteristics are all derivatives of a single benzene ring, we believe such a strategy provides a rational and efficient approach for the fabrication of novel organic solid fluorophores from both fundamental and practical viewpoints.

\section{Acknowledgements}

We are grateful for financial support from the National Natural Science Foundation of China (21402115, 51403122 and 21672135), the Fundamental Research Funds for the Central Universities (GK201503028) and the Natural Science Foundation of Shaanxi Province (2015JQ5150 and 2016JQ2020). We also appreciate Dr F. Y. Liu and Dr J. Liu for helpful discussions on theoretical calculations and DLS analysis.

\section{Notes and references}

1 (a) L. Duan, J. Qiao, Y. Sun and Y. Qiu, Adv. Mater., 2011, 23, 1137-1144; (b) R. H. Friend, R. W. Gymer, A. B. Holms, J. H. Burroughes, R. N. Marks, C. Taliani, D. D. C. Bradley, D. A. Dos Santos, J. L. Brédas, M. Lögdlund and W. R. Salaneck, Nature, 1999, 397, 121-128; (c) A. C. Grimsdale, K. L. Chan, R. E. Martin, P. G. Jokisz and A. B. Holmes, Chem. Rev., 2009, 109, 897-1091; (d) T. Qin, W. Wiedemair, S. Nau, R. Trattnig, S. Sax, S. Winkler, A. Vollmer, N. Koch, M. Baumgarten, E. J. W. List and K. Müllen, J. Am. Chem. Soc., 2011, 133, 1301-1303.

2 J. Zhang, W. Chen, J. A. Rojas, V. E. Jucov, V. T. Timofeeva, C. T. Parker, S. Barlow and R. S. Marder, J. Am. Chem. Soc., 2013, 135, 16376-16379.

3 T. Weil, T. Vosch, J. Hofkens, K. Peneva and K. Müllen, Angew. Chem., Int. Ed., 2010, 49, 9068-9093.

4 (a) S. W. Thomas III, G. D. Joly and T. M. Swager, Chem. Rev., 2007, 107, 1339-1386; (b) A. P. de Silva, H. Q. N. Gunaratne, T. Gunnlaugsson, A. J. M. Huxley, C. P. McCoy, J. T. Rademacher and T. E. Rice, Chem. Rev., 1997, 97, 1515-1566; (c) L. Basabe-Desmonts, D. N. Reinhoudt and M. Crego-Calama, Chem. Soc. Rev., 2007, 36, 993-1017.

5 (a) G. S. Beddard and G. Porter, Nature, 1976, 260, 366-367; (b) Y. Hong, J. W. Y. Lam and B. Z. Tang, Chem. Commun., 2009, 4332-4353; (c) C.-K. Lim, S. Kim, I. C. Kwon, C.-H. Ahn and S. Y. Park, Chem. Mater., 2009, 21, 5819-5825.

6 (a) J. Luo, Z. Xie, J. W. Y. Lam, L. Cheng, H. Chen, C. Qiu, H. S. Kwok, X. Zhan, Y. Liu, D. Zhu and B. Z. Tang, Chem. Commun., 2001, 1740-1741; (b) B.-K. An, S.-K. Kwon, S.-D. Jung and S. Y. Park, J. Am. Chem. Soc., 2002, 124, 14410-14415.

7 (a) J. Chen, C. C. W. Law, J. W. Y. Lam, Y. Dong, S. M. F. Lo, I. D. Williams, D. Zhu and B. Z. Tang, Chem. Mater., 2003, 15, 1535-1546; (b) G. Yu, S. Yin, Y. Liu, J. Chen, X. Xu, X. Sun, D. Ma, X. Zhan, Q. Peng, Z. G. Shuai, B. Z. Tang, D. B. Zhu, W. Fang and Y. Luo, J. Am. Chem. Soc., 2005, 127, 63356346; (c) J. Mei, Y. Hong, J. W. Y. Lam, A. Qin and B. Z. Tang, Adv. Mater., 2014, 26, 5429-5479.

8 (a) J. Mei, N. L. C. Leung, R. T. K. Kwok, J. W. Y. Law and B. Z. Tang, Chem. Rev., 2015, 115, 11718-11940; (b) S. Xu, T. Liu, Y. Mu, Y. F. Wang, Z. Chi, C. C. Lo, S. Liu, Y. Zhang, A. Lien and J. Xu, Angew. Chem., Int. Ed., 2015, 54, 874-878; (c) S.-J. Yoon, J. W. Chung, J. Gierschner, K. S. Kim, M.-G. Choi, D. Kim and S. Y. Park, J. Am. Chem. Soc., 2010, 132, 13675-13683; (d) Y. Okazawa, K. Kondo, M. Akita and M. Yoshizawa, J. Am. Chem. Soc., 2015, 137, 98-101; (e) T. Shiragami, Y. Nakamura, J. Matsumoto, M. Otsuki and M. Yasuda, Phys. Chem. Chem. Phys., 2014, 16, 22046-22051; (f) X. Wang, J. Hu, G. Zhang and S. Liu, J. Am. Chem. Soc., 2014, 136, 9890-9893; $(g)$ J. Zhang, B. Xu, J. Chen, S. Ma, Y. Dong, L. Wang, B. Li, L. Ye and W. Tian, Adv. Mater., 2014, 26, 739-745; (h) R. Yoshii, A. Hirose, K. Tanaka and Y. Chujo, J. Am. Chem. Soc., 2014, 136, 18131-18139; (i) S. Xu, Y. Yuan, X. Cai, C. J. Zhang, F. Hu, J. Liang, G. Zhang, D. Q. Zhang and B. Liu, Chem. Sci., 
2015, 6, 5824-5830; (j) L. Peng, Y. Zheng, X. Wang, A. J. Tong and Y. Xiang, Chem.-Eur. J., 2015, 21, 4326-4332; (k) J. Huang, N. Sun, Y. Dong, R. Tang, P. Lu, P. Cai, Q. Q. Li, D. Ma, J. Qin and Z. Li, Adv. Funct. Mater., 2013, 23, 23292337; (l) G. Chen, W. Li, T. Zhou, Q. Peng, D. Zhai, H. Li, W. Z. Yuan, Y. M. Zhang and B. Z. Tang, Adv. Mater., 2015, 27, 4496-4501; (m) M. Huang, R. Yu, K. Xu, S. Ye, S. Kuang, X. Zhu and Y. Wan, Chem. Sci., 2016, 7, 4485-4491.

9 (a) Z. Zhang, B. Xu, J. Su, L. Shen, Y. Xie and H. Tian, Angew. Chem., Int. Ed., 2011, 50, 11654-11657; (b) S. P. Anthony, ChemPlusChem, 2012, 77, 518-531.

10 (a) Z. R. Grabowski, K. Rotkiewicz and W. Rettig, Chem. Rev., 2003, 103, 3899-4032; (b) C. T. Chen, Chem. Mater., 2004, 16, 4389-4400.

11 (a) A. Wakamiya, K. Mori and S. Yamaguchi, Angew. Chem., Int. Ed., 2007, 46, 4273-4276; (b) M. Shimizu, Y. Takeda, M. Higashi and T. Hiyama, Angew. Chem., Int. Ed., 2009, 48, 3653-3656; (c) E. Kim, Y. Lee, S. Lee and S. B. Park, Acc. Chem. Res., 2015, 48, 538-547; (d) A. C. Shaikh, D. S. Ranade, S. Thorat, A. Maity, P. P. Kulkarni, R. G. Gonnade, P. Munshi and N. T. Patil, Chem. Commun., 2015, 51, 16115-16118; (e) Z. Song, W. Zhang, M. Jiang, H. H. Y. Sung, R. T. K. Kwok, H. Nie, I. D. Williams, B. Liu and B. Z. Tang, Adv. Funct. Mater., 2016, 26, 824-832.

12 (a) K. A. N. Upamali, L. A. Estrada, P. K. De, X. Cai, J. A. Krause and D. C. Neckers, Langmuir, 2011, 27, 15731580; (b) B.-K. An, J. Gierschner and S. Y. Park, Acc. Chem.
Res., 2012, 45, 544-554; (c) E. Ishow, A. Brosseau, G. Clavier, K. Nakatani, P. Tauc, C. Fiorini-Debuisschert, S. Neveu, O. Sandre and A. Léaustic, Chem. Mater., 2008, 20, 6597-6599.

13 CCDC 1056239 (1a), 1056280 (1c), 1403124 (1d), 1439334 (2a), 1439332 (2b), 1443616 (2c), 1472574 (2d-g), 1499316 (2d-o), 1442948 (2e) and 1442404 (2g) contain the ESI crystallographic data for this paper. $\dagger$

14 The solvent effects with 2 f could not be measured due to its poor solubility.

15 M. D. Curtis, J. Cao and J. W. Kampf, J. Am. Chem. Soc., 2004, 126, 4318-4328.

16 (a) Z. He, L. Zhang, J. Mei, T. Zhang, J. W. Y. Lam, Z. Shuai, Y. Q. Dong and B. Z. Tang, Chem. Mater., 2015, 27, 66016607; (b) Y. Sagara and T. Kato, Nat. Chem., 2009, 1, 605610; (c) K. Nagura, S. Saito, H. Yusa, H. Yamawaki, H. Fujihisa, H. Sato, Y. Shimoikeda and S. Yamaguchi, J. Am. Chem. Soc., 2013, 135, 10322-10325; (d) C. Dou, L. Han, S. Zhao, H. Zhang and Y. Wang, J. Phys. Chem. Lett., 2011, 2, 666-670; (e) H. Naito, Y. Morisaki and Y. Chujo, Angew. Chem., Int. Ed., 2015, 54, 5084-5087; (f) M.-S. Yuan, D.-E. Wang, P. Xue, W. Wang, J.-C. Wang, Q. Tu, Z. Liu, Y. Liu, Y. Zhang and J. Wang, Chem. Mater., 2014, 26, 2467-2477; (g) Z. Chi, X. Zhang, B. Xu, X. Zhou, C. Ma, Y. Zhang, S. Liu and J. Xu, Chem. Soc. Rev., 2012, 41, 3878-3896. 